\title{
Successful use of a topical mixture with ozolipoile in the treatment of actinic ulcers
}

This article was published in the following Dove Press journal:

Clinical, Cosmetic and Investigational Dermatology

I April 2015

Number of times this article has been viewed

\section{Francesco Inchingolo' \\ Angelo Tarullo ${ }^{2}$ \\ Raffaele Cagiano ${ }^{2}$ \\ Gianpiero Resta ${ }^{3}$ \\ Gianna Dipalma ${ }^{3}$ \\ Angelo Michele Inchingolo 3 \\ Antonella Tarullo ${ }^{3}$ \\ Salvatore Scacco ${ }^{4}$ \\ Massimo Marrelli ${ }^{5,6, *}$ \\ Luigi Corti ${ }^{7}, *$ \\ Marco Tatullo 8 ,*}

'Department of Interdisciplinary Medicine, University of Bari "Aldo

Moro", Bari, 'Department of

Biomedical Sciences and Human

Oncology, University of Bari "Aldo

Moro", Bari, "3Private Practice, Dental

Clinic, Bari, " ${ }^{4}$ epartment of Basic

Medical Sciences, Neurosciences and

Sensorial Organs, University of Bari

"Aldo Moro", Bari, ${ }^{5}$ Maxillofacial

Unit, Calabrodental Clinic, Crotone,

${ }^{6} \mathrm{Healthcare}$ Center, Marrelli Hospital,

Crotone, ${ }^{7} \mathrm{UOC}$ of Radiotherapy and Nuclear Medicine IOV - IRCCS,

University of Padova, Padova,

${ }^{8}$ Biomedical Section, Tecnologica

Research Institute, Crotone, Italy

*These authors equally contributed to this research paper

Correspondence: Francesco Inchingolo Department of Interdisciplinary Medicine, University of Bari “Aldo Moro", P.ce Giulio Cesare, General Hospital, Bari, Italy

Tel +3933 I21। II04

Email prof.inchingolo@libero.it
Purpose: Post-radiation skin damage is the result of alterations produced in the irradiated zone. There are different studies aimed at verifying the effectiveness of several kinds of molecules in the treatment of radiation-induced skin damage. The purpose of this study is to test a mixture with a formulation containing several natural active ingredients on actinic ulcers in patients receiving radiation therapies.

Patients and methods: The authors recruited 13 patients, and randomly divided them into a test group $\left(\mathrm{T}_{1}\right)$ and a control group $\left(\mathrm{T}_{2}\right)$. The patients in both groups were undergoing radiotherapy. The $T_{1}$ group was treated with a mixture called ozolipoile, whereas the $T_{2}$ group was administered hyaluronic acid gel followed by non-ablative laser therapy. We evaluated the obtained results, the time of clinical healing, the reduction of pain, and each side effect, comparing all data between the $\mathrm{T}_{1}$ and $\mathrm{T}_{2}$ groups.

Results: The average Visual Analog Scale results showed decrease in pain in both groups; however, while the $T_{1}$ group showed a significant decrease in the values, the $T_{2}$ group reported a more gradual reduction in the values, without ever reaching the minimum values obtained with the treatment with ozolipoile.

Conclusion: Treatment of actinic ulcers with ozolipoile mixture leads to faster control of pain and to better healing of small-size ulcers.

Keywords: actinic ulcers, radiation-induced ulcers, skin wound, ozonide, wound healing

\section{Introduction}

Chronic skin lesions show a low tendency to spontaneous healing. ${ }^{1}$ Chronic injuries are particularly painful and debilitating for the patient, and they are often the manifestation of an underlying condition with a vascular etiopathogenesis that obstructs healing. These factors cause a slowing of the reconstruction processes of the matrix and of the reepithelialization, favoring, consequently, a process of necrosis.

Chronic injuries are represented by ischemic ulcers, diabetic ulcers, venous ulcers, and pressure ulcers that do not reepithelialize. Post-radiation skin damage is the result of alterations produced in the irradiated zone, although the major site of damage is the germinal layer of the epidermis and the early response is clearly evident in the capillary network of the dermis: the dilation of these capillaries and the release of histamine causes a characteristic skin erythema. ${ }^{2}$ After this transient erythema, a latency period follows. Even this second phase is due to radiation-induced capillary dilation: the capillaries dilate to compensate the reduced oxygen supply due to the peripheral ischemia of the arterioles. The second phase of erythema is accompanied by the loss of the superficial layers of the epidermis: the skin appears similar to that observed in 
first-degree thermal burns. The radiation-induced death of the germinative layer of the epidermis causes an extended desquamation. ${ }^{2,3}$

There are different studies aimed at verifying the effectiveness of several kinds of molecules in the treatment of radiation-induced skin damages, and some of them have, in fact, produced good results by using biomolecules rich in oxygen, ${ }^{4,5}$ thanks to the hyperoxygenation of the tissues. These biomolecules promote the healing of skin wounds and of mucous membranes, regenerating them and forming a new epithelial layer. ${ }^{6}$

In addition, they also act as antibacterial and antifungal agents due to their oxidizing action. ${ }^{7}$

Other studies tested, instead, alpha-tocopheryl acetate, to prove its radical scavenging activity: ${ }^{8}$ this liposoluble vitamin blocks the lipid peroxidation of membranes; therefore, it contrasts the free radical species responsible for cell aging and for the damages from excessive exposure to solar radiation. A well-known category of molecules used in the treatment of skin damages is ozonides. ${ }^{9}$ They act on biochemical mediators and enzymes involved in the mechanism of inflammation by activating defense systems.

These different pharmacologically active molecules have been widely tested individually, but there is not much evidence of their synergistic action.

The purpose of this study is to test a mixture with a formulation containing all the active ingredients described above, together with other natural ingredients, to determine whether they, in a single solution, are able to add up their beneficial effects on ulcerated skin tissues in patients receiving radiation therapies.

\section{Material and methods}

The authors recruited 13 patients, all female, aged from 62 years to 65 years, from private studies mainly located in south Italy. These patients were recruited randomly, were clearly informed regarding the study, and signed an informed consent. The study was conducted in compliance with the "ethical principles for medical research involving human subjects" of the Declaration of Helsinki. The study was conducted in accordance with Italian laws and regulations.

The enrolled patients suffered from second- or thirddegree actinic ulcers following a radiotherapy cycle, according to the EORTC classification. ${ }^{10}$

All the patients were suffering from head and neck oncological pathology.

These patients were randomly divided into a test group $\left(\mathrm{T}_{1}\right)$ and a control group $\left(\mathrm{T}_{2}\right)$. The $\mathrm{T}_{1}$ group was treated

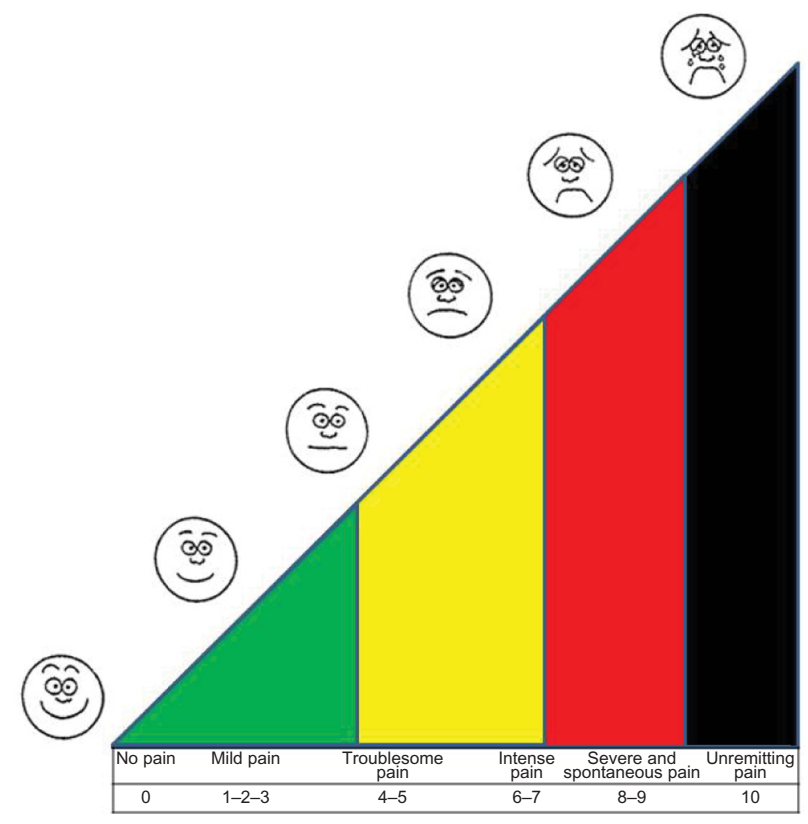

Figure I VAS (Visual Analog Scale) for the monitoring of pain.

with a mixture called ozolipoile: the treatment tested was a medicament mainly composed of ozone, oleic acid, $\alpha$-lipoic acid, vitamin $\mathrm{E}$ acetate, panthenol, tocopheryl acetate, and tocopherol. Ozolipoile is patented and already marketed; thus, no experimental studies were carried out on the patients, but we have assessed the efficacy of this treatment.

Instead, the $\mathrm{T}_{2}$ group was administered hyaluronic acid gel followed by non-ablative laser therapy, as previously described. ${ }^{11}$

At the enrollment phase, each patient received exhaustive information about personal hygiene and the mode of application of the mixture, along with a card to express numerically the level of pain the patient was suffering according to a Visual Analog Scale (VAS), ranging from 0 (no pain) to 10 (unremitting pain) (Figure 1). Both the protocols should be administered about three times a day, at least 2 hours before and after the radiotherapy, until complete healing. A clinical measurement was performed and archived before the start of the protocols (Table 1). Sterile protective dressings of ulcers were admitted. Clinical observations of the patients were per-

Table I Baseline data related to the ulcerate in $T_{1}$ and $T_{2}$ groups

\begin{tabular}{lll}
\hline Baseline data & $\mathbf{T}_{1}$ group & $\mathbf{T}_{2}$ group \\
\cline { 2 - 3 } & Mean & Mean \\
\hline Area of the ulcer $(\mathrm{cm})$ & 3.8 & 4.1 \\
Duration in days & 5 & 7 \\
\hline
\end{tabular}

Notes: $T_{1}$, test group treated with a mixture called ozolipoile; $T_{2}$, control group treated with a combined administration of hyaluronic acid gel followed by a nonablative laser/radiofrequency/intense pulsed light therapy. 
Table 2 Synoptical table comparing the TI and T2 VAS scores

VAS scores

\begin{tabular}{llllll}
\hline Baseline Day I Day 3 & Day 5 & Day 7 & DI-D7
\end{tabular}

variation

\begin{tabular}{|c|c|c|c|c|c|c|}
\hline \multicolumn{7}{|c|}{ Group $T_{1}$} \\
\hline Median & $9.0 \pm 0.5$ & $7 \pm 1.5$ & $5 \pm 0.5$ & $2.5 \pm 0.5$ & $I \pm 1$ & $92.7 \%$ \\
\hline Mean & & 8.3 & 5.3 & 2.5 & 0.5 & 7.7 \\
\hline \multicolumn{7}{|c|}{ Group $T_{2}$} \\
\hline Median & $9.5 \pm 0.5$ & $7 \pm 1$ & $6.5 \pm 1.5$ & $3.5 \pm 1.5$ & $2 \pm 1$ & $67.5 \%$ \\
\hline Mean & & 7.7 & 6.5 & 3.5 & 2.5 & 5.2 \\
\hline
\end{tabular}

Notes: $T_{1}$, test group treated with a mixture called ozolipoile; $T_{2}$, control group treated with a combined administration of hyaluronic acid gel followed by a nonablative laser/RF/IPL therapy.

Abbreviations: VAS, Visual Analog Scale; RF, radiofrequency; IPL, intense pulsed light.

formed daily during radiotherapy and the data were collected at days 1,3,5, and 7 from the beginning. The follow-up was aimed at registering any changes in the perception of pain related to the eventual healing process of the ulcers. In order to not alter the collected data, each patient was monitored by the same physician, who scored the data without being aware of the difference between the treatments of the $T_{1}$ and $\mathrm{T}_{2}$ groups. We evaluated the obtained results, the time of clinical healing, the reduction in pain, and each side effect, comparing all data between the $\mathrm{T}_{1}$ and $\mathrm{T}_{2}$ groups.

\section{Results}

The results carried out from VAS showed a significant difference in the results of the treatments of the $T_{1}$ and $T_{2}$ groups (Table 2). The first day of treatment achieved best scores in the $T_{2}$ group: despite the pain increasing immediately after the application of the laser beam, because of the vascular effect depending on the local heat, at the end of treatment, the patients of this group referred reduced pain in the treated area. However, by the third day of treatment, the VAS scores gave an average of 5.3 in $_{1}$, instead of 6.5 in $\mathrm{T}_{2}$.

The average VAS results showed a decrease in pain in both groups; however, while the $\mathrm{T}_{1}$ group showed a significant decrease in the values between day 1 and day 7 , the $\mathrm{T}_{2}$ group reported a more gradual reduction in the values, without ever reaching the minimum values obtained with the treatment with ozolipoile.

In all reported cases, scars of different degrees appeared at the end of radiotherapy, even if only 7 days from the beginning of $\mathrm{T}_{1}$ treatment, the ulcers appeared less deep and reduced in size (Figure 2). The small-size ulcers were fully healed within 3-5 days; in those cases esthetically more compromised, plastic surgery has been performed with minimally invasive techniques. ${ }^{12}$

\section{Discussion}

In second-degree radiodermatitis, erythema usually occurs very early and is accompanied by intense edema and by the formation of blisters and bubbles. On the fifteenth day after the irradiation, it appeared as a richly vascularized area, and this form was associated with intense pain; repairing of the injuries occurred in a few months, but usually functional alterations of the skin, telangiectasia, and skin xeroderma remained. Ulcerative radiodermatitis usually shows no signs of repair, and in the most favorable cases, the healing is slow, with frequent recurrences. ${ }^{1-3}$

The treatment of radiation-induced ulcers is initially aimed at the removal of necrotic tissue, the elimination of bacteria, and the drainage of infected lesions..$^{13,14}$

Reconstructive surgery could be indicated in areas deeply atrophic, following the radiotherapy. ${ }^{15,16}$

Local treatment with detergent and cleaning compounds is based on the use of alkaline solutions: it is advisable to perform all those measures addressed to obtain a gradual cleansing and the development of granulation tissue. Our treatment has shown very good performance not only on small-size ulcers but also on those wider and on the surrounding tissues: the combination of active substances against inflammation, the oxidative killing by the ozonides, the massive action against free radicals carried out by antioxidants, and the barrier effect

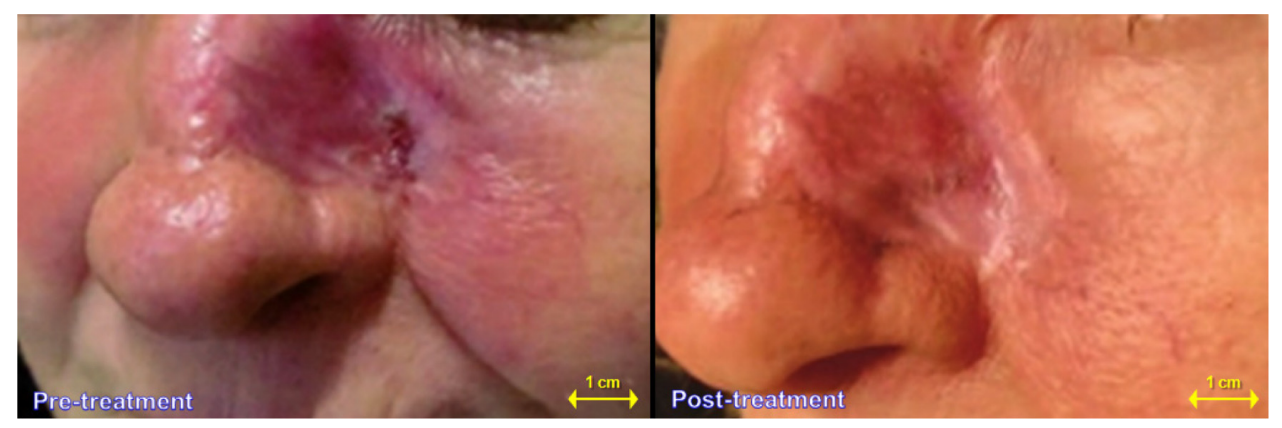

Figure 2 Actinic lesion with a wide erythematous area: a case-control study. The same case-control study 7 days after beginning of $T$, treatment. 
by alpha-lipoic acid have created the best conditions for this impressive outcome in our first pilot study.

\section{Conclusion}

In conclusion, the treatments of actinic ulcers based on ozolipoile mixture show important advantages in controlling pain and healing time. The same procedure could be extended to chronic skin ulcers of different etiologies, given that the skin damage is similar, and in the most favorable cases, this treatment may even result more effective than in actinic ulcers.

\section{Disclosure}

The authors report no conflicts of interest in this work.

\section{References}

1. Häkkinen L, Koivisto L, Gardner H, et al. Increased expression of beta6integrin in skin leads to spontaneous development of chronic wounds. Am J Pathol. 2004;164(1):229-242.

2. Goessler UR, Bugert P, Kassner S, et al. In vitro analysis of radiationinduced dermal wounds. Otolaryngol Head Neck Surg. 2010;142(6): $845-850$.

3. Olascoaga A, Vilar-Compte D, Poitevin-Chacón A, Contreras-Ruiz J. Wound healing in radiated skin: pathophysiology and treatment options. Int Wound J. 2008;5(2):246-257. [Review].

4. Mills BJ. Wound healing: the evidence for hyperbaric oxygen therapy. Br J Nurs. 2012;21(20):28, 30, 32, 34.

5. González-Espinosa D, Pérez-Romano L, Guzmán-Soriano B, Arias E, Bongiovanni CM, Gutiérrez AA. Effects of pH-neutral, super-oxidised solution on human dermal fibroblasts in vitro. Int Wound J. 2007;4(3): 241-250.
6. Bhutani S, Vishwanath G. Hyperbaric oxygen and wound healing. Indian J Plast Surg. 2012;45(2):316-324.

7. Cimșit M, Uzun G, Yildiz S. Hyperbaric oxygen therapy as an antiinfective agent. Expert Rev Anti Infect Ther. 2009; 7(8):1015-1026.

8. Hara H, Sukamoto T, Ohtaka H, et al. Effects of baicalein and alphatocopherol on lipid peroxidation, free radical scavenging activity and 12-O-tetradecanoylphorbol acetate-induced ear edema. Eur $J$ Pharmacol. 1992;221(2-3):193-198.

9. Reyes JG, Lanes J. Treatment of leg ulcers with ozonide of olive oil in ointment form. Urol Cutaneous Rev. 1947;51(10):592-597.

10. Hoeller U, Tribius S, Kuhlmey A, Grader K, Fehlauer F, Alberti W. Increasing the rate of late toxicity by changing the score? A comparison of RTOG/EORTC and LENT/SOMA scores. Int J Radiat Oncol Biol Phys. 2003;55:1013-1018.

11. Goldman MP, Alster TS, Weiss R. A randomized trial to determine the influence of laser therapy, monopolar radiofrequency treatment, and intense pulsed light therapy administered immediately after hyaluronic acid gel implantation. Dermatol Surg. 2007;33(5):535-542.

12. Inchingolo F, Tatullo M, Abenavoli FM, et al. Surgical treatment of depressed scar: a simple technique. Int J Med Sci. 2011;8(5):377-379.

13. Heilborn JD, Weber G, Grönberg A, Dieterich C, Ståhle M. Topical treatment with the vitamin $\mathrm{D}$ analogue calcipotriol enhances the upregulation of the antimicrobial protein hCAP18/LL-37 during wounding in human skin in vivo. Exp Dermatol. 2010;19(4):332-338.

14. Janković A, Binić I, Vručinić Z, Janković D, Janković I, Jančić S. Can you combine herbal therapy with physical agents in the treatment of venous leg ulcers? Evaluation of healing and antimicrobiological effects: a pilot study. Forsch Komplementmed. 2010;17(5):266-269.

15. Inchingolo F, Tatullo M, Pacifici A, et al. Use of dermal-fat grafts in the post-oncological reconstructive surgery of atrophies in the zygomatic region: clinical evaluations in the patients undergone to previous radiation therapy. Head Face Med. 2012;8:33. [Review].

16. Inchingolo F, Tatullo M, Abenavoli FM, et al. Simple technique for augmentation of the facial soft tissue. Scientific World Journal. 2012;2012:262989.
Clinical, Cosmetic and Investigational Dermatology

\section{Publish your work in this journal}

Clinical, Cosmetic and Investigational Dermatology is an international, peer-reviewed, open access, online journal that focuses on the latest clinical and experimental research in all aspects of skin disease and cosmetic interventions. All areas of dermatology will be covered; contributions will be welcomed from all clinicians and

\section{Dovepress}

basic science researchers globally. This journal is indexed on CAS The manuscript management system is completely online and includes a very quick and fair peer-review system, which is all easy to use. Visit http://www.dovepress.com/testimonials.php to read real quotes from published authors. 